\title{
PANTON-VALENTINE LEUKOCIDIN AND STAPHYLOCOCCAL CASSETTE CHROMOSOME MEC CHARACTERIZATION OF COMMUNITY ACQUIRED METHICILLIN-RESISTANT STAPHYLOCOCCUS AUREUS
}

\author{
Velma Rebić1, Ana Budimir², Mufida Aljicević1, Sabina Mahmutović Vranić1, Damir Rebić \\ ${ }^{1}$ Institute of Microbiology, Faculty of Medicine, University of Sarajevo, Sarajevo, Bosnia and Herzegovina \\ ${ }^{2}$ Department of Clinical and Molecular Microbiology, University Hospital Centre Zagreb, Zagreb, Croatia \\ ${ }^{3}$ Clinic for Nephrology, University Clinical Centre of Sarajevo, Sarajevo, Bosnia and Herzegovina
}

\section{SUMMARY}

Objectives: Staphylococcus aureus (SA) represents one of the most important microorganism that is part of the normal microflora of humans, but in certain conditions can cause very serious infections. Methicillin-resistant Staphylococcus aureus (MRSA) is responsible for a wide spectrum of nosocomial and community associated infections worldwide. The aim of this study was to determine community acquired MRSA (CA-MRSA), as well as the frequency of Panton-Valentine leukocidin (PVL) genes and staphylococcal cassette chromosome mec (SCCmec) types in isolates obtained from outpatients in the region of 700,000 people (Canton Sarajevo, Bosnia and Herzegovina)

Methods: Our investigation included phenotypic and genotypic markers such as antimicrobial resistance, pulsed-field gel electrophoresis (PFGE), SCC typing, and PVL detection.

Results: Antimicrobial susceptibility: all MRSA isolates were resistant to the $\beta$-lactam antibiotics tested, and all isolates were susceptible to trimethoprim sulphamethoxazole, rifampicin, fusidic acid, linezolid, and vancomycin. After the PFGE analysis, the isolates were grouped into five similarity groups: A-E. The largest number of isolates belonged to one of two groups: $C-60 \%$ and $D-27 \%$. In both groups $C$ and D, SCCmec type IV was predominant ( $60 \%$ and $88.8 \%$, respectively). A total of $24 \%$ of the isolates had positive expression of PVL genes, while $76 \%$ showed a statistically significantly greater negative expression of PVL genes.

Conclusions: Using combination techniques, we were able to investigate the origin and genetic background of the strains. PFGE analysis revealed two large, genetically related groups of strains consisting of 87 isolates. Our results suggest failure to apply the screening policy, and a lack of knowledge about multiresistant MRSA strains. This study showed the local epidemiological situation which should be the basis of antimicrobial empiric therapy for non-hospitalized patients.

Key words: methicillin resistance, Panton-Valentine leukocidin, outpatients, types

Address for correspondence: V. Rebić, Institute of Microbiology, Faculty of Medicine, University of Sarajevo, Čekaluša 90, 71000 Sarajevo, Bosnia and Herzegovina. E-mail: velmarebic@yahoo.com

https://doi.org/10.21101/cejph.a4979

\section{INTRODUCTION}

Staphylococcus aureus (SA) represents one of the most important microorganism that is part of the normal microflora of humans, but in certain conditions can cause very serious infections. Approximately $20-30 \%$ of the general human population is persistently colonized with SA (1). Staphylococcus normally causes a spectrum of infections ranging from common, mild skin and soft tissue infections to hematogenous infection with multiorgan injury. Staphylococcus aureus is notorious for its ability to become resistant to antibiotics, therefore, treatment of these infections has become problematic due to the development of resistance, especially methicillin-resistant SA (2).

Methicillin-resistant SA (MRSA) includes those strains that have acquired a gene giving them resistance to methicillin and essentially all other beta-lactam antibiotics. MRSA was first reported in 1961, soon after methicillin was introduced into human medicine to treat penicillin-resistant staphylococci. This group of organisms has since emerged as a serious concern in human medicine (3). MRSA was first reported as a hospital-acquired pathogen in human hospitals (4).

During the past four decades, MRSA is largely a hospital-acquired (HA) infection, in fact, one of the most common. Recently, new strains have emerged in the communities that are capable of causing severe infections in otherwise healthy people. These strains are known as community-acquired methicillin-resistant SA (CA-MRSA) (4). By using molecular and epidemiological techniques, it became evident that CA-MRSA strains have genetic and phenotypic characteristics that are different from those of HA-MRSA. CA-MRSA tends to be susceptible to a variety 
of non- $\beta$-lactam antibiotics, whereas HA-MRSA are typically multiresistant to antibiotics.

The resistance of SA to beta-lactam antibiotics is associated with an expression of penicillin-binding protein $2 \mathrm{a}(\mathrm{PBP} 2 \mathrm{a})$. This protein is encoded by the mecA gene, which is situated on a mobile genetic element, staphylococcal cassette chromosome mec (SCCmec) (5). So far, there are 11 known and described types of SCCmec. Based on SCCmec typing, HA-MRSA usually carry SCCmec I, II, and III, while CA-MRSA strains typically harbour SCCmec types IV and V, as many other types (VI-XI). SCCmec types IV and V are smaller in size compared to SCCmec types I, II and III (6).

In addition, CA-MRSA are more likely than HA-MRSA isolates to encode a putative virulence factor, i.e. Panton-Valentine leukocidin (PVL). Panton-Valentine leukocidin is a pore-forming bicomponent cytotoxin that results in leukocyte lysis. The toxin is encoded by two genes, $l u k S-P V$ and $l u k F-P V$, which are carried on a bacteriophage that has incorporated itself into the $S$. aureus chromosome. It is capable of destroying human leukocytes and inflicting severe tissue damage. PVL may be the key toxin responsible for enhanced virulence of CA-MRSA, but this is controversial (7).

Although the direct role of PVL in the pathogenesis of CA-MRSA-mediated infections is unclear, several studies have reported an association between PVL and skin and soft-tissue infections, necrotizing pneumonia, and osteomyelitis $(8,9)$.

The aim of this study was to determine CA-MRSA, as well as the frequency of PVL genes and staphylococcal cassette chromosome mec ( $\mathrm{SCCmec}$ ) types in isolates obtained from outpatients in the region of 700,000 people. They all belong to the Microbiology Laboratory of the Institute of Public Health of the Canton Sarajevo, Bosnia and Herzegovina.

\section{MATERIALS AND METHODS}

During the 6-month period, a total of 2,279 Staphylococcus aureus strains were isolated from the patient samples of patients visiting the Laboratory of the Institute of Public Health of Canton Sarajevo, Bosnia and Herzegovina.

The strains were recovered from different samples including nose swabs, throat, ear, eye, umbilicus swabs, wound and skin swabs. Swabs were streaked onto sheep blood agar (5\% Columbia agar base) (Oxoid Microbiology Products, UK) for detection of gram-positive bacteria and incubated overnight at $37^{\circ} \mathrm{C}$. Colonies with typical morphology were identified by standard microbiological tests such as catalase, tube coagulase, mannitol fermentation, and DNase.

The isolates were tested for methicillin resistance by disc diffusion (DD) test at Mueller-Hinton (MH) (Oxoid Microbiology Products (UK, CM0337) agar using oxacillin $(1 \mu \mathrm{g})$ and cefoxitin $(30 \mu \mathrm{g})$ according to the Clinical and Laboratory Standards Institute (CLSI) guidelines (September 2018). The plates were incubated at $37^{\circ} \mathrm{C}$ for 18 hours and zones of inhibition were measured. An inhibition zone of $\geq 13 \mathrm{~mm}$ was considered susceptible, $11-12 \mathrm{~mm}$ was considered intermediate and $\leq 10 \mathrm{~mm}$ resistant for oxacillin. An inhibition zone of $\geq 20 \mathrm{~mm}$ was considered susceptible and $\leq 19 \mathrm{~mm}$ resistant for cefoxitin.

Further they have been tested by phenotypic methods for MRSA strains. The following methods were used: E-test MIC, chromID MRSA test and PBP2a latex agglutination test.

\section{E-test}

Minimal inhibitory concentrations (MICs) were determined with the E-test system (BioMerieux, Marcy L'Etoile, France) according to the manufacturer's instructions. Briefly, MuellerHinton agar plate supplemented with $2 \% \mathrm{NaCl}$ was used. An inoculum of SA isolates was applied and an oxacillin (Ox) E-test strip having a gradient range of $0.016-256 \mu \mathrm{g} / \mathrm{ml}$ was placed on the plate. After 24 hours incubation at $35-37^{\circ} \mathrm{C}$ in ambient air, the plates were read using a transmitted light source. MIC of $2 \mu \mathrm{g} / \mathrm{ml}$ were considered susceptible and 4 $\mu \mathrm{g} / \mathrm{ml}$ resistant.

\section{ChromID MRSA Test}

ChromID MRSA test (bioMérieux, France) is a chromogenic medium comprised of a rich nutritive base of different peptones and a combination of antibiotics, including cefoxitin for the selection of growth of MRSA. Activity of the $\alpha$-glukosidase by MRSA produces green-coloured colonies. Inoculated plates are incubated aerobically between $35^{\circ} \mathrm{C}$ and $37^{\circ} \mathrm{C}$, while minimizing exposure to light. The plates are approved to be examined after 24 hours and if any green-coloured colonies are observed, no confirmatory test is needed for the identification of MRSA.

\section{PBP2a Latex Agglutination Test}

The Slidex MRSA Detection (Biomérieux, Paris, France), which is based on the agglutination of latex particles sensitized with monoclonal antibodies against PBP2a, was carried out and interpreted according to the manufacturer's instructions.

\section{Antibiotic Susceptibility Testing}

All MRSA isolates were tested for antimicrobial susceptibility through disk diffusion method (Kirby-Bauer), according to the Clinical and Laboratory Standards Institute (CLSI) guidelines. Susceptibility was tested to a panel of 12 antibiotics: oxacillin, cefoxitin, erythromycin, clindamycin, linezolid, ciprofloxacin, sulfamethoxazole-trimethoprim, tetracycline, fusidic acid, rifampicin, vancomycin, and gentamicin. In case of fusidic acid, the European Committee on Antimicrobial Susceptibility Testing (EUCAST) guidelines from 2018 were used.

We selected a representative sample of 100 strains, eliminating the "copy" strains (strains from the same patient at different places and those that are repeated) randomly trying to follow the dynamics of equal representation of different months study period. The isolates were stored at $-70^{\circ} \mathrm{C}$ and transported to the Department of Clinical and Molecular Microbiology, University Hospital Centre Zagreb, Croatia, where they were analyzed by molecular methods.

\section{Molecular Analysis}

Molecular analysis of the SCCmec cassette was performed using a method previously described by Oliveira and de Lencastre (10), with certain modification described by Budimir et al. (11). PVL was detected using PCR primers previously described by Lina et al. (12). PFGE was performed as described in the study by Tenover et al. with some adjustments $(13,14)$. 
The band patterns were analyzed with Dice comparison and unweighted-pair group matching analysis settings with GelCompar II (Applied Maths, Sint-Martens-Latem, Belgium) according to the scheme by Tenover et al. (14). Relatedness of $80 \%$ or more was considered significant, and strains with at least $80 \%$ similarity were considered to be related to each other and were grouped together in a similarity group or genotype.

Spa typing was performed according to previously published methods $(15,16)$. An analysis of sequence chromatograms was performed using Ridom Staph Type (Ridom GmbH, Wurzburg, Germany).

\section{Statistical Analysis}

All data were expressed as the mean SD or as median and interquartile range. The distribution of variables was tested by the Kolmogorov-Smirnov test. For the analysis of categorical variables was used chi-square test or Fisher test. The difference between variables was analyzed by the Mann-Whitney test. P-values less than 0.05 were considered statistically significant. All statistical calculations were performed with the SPSS 16 software (version 16.0, SPSS Inc., Chicago, IL, USA).

\section{RESULTS}

Out of 2,279 Staphylococcus aureus strains, 653 were methicillin-resistant Staphylococcus aureus. A total of 653 methicillin-resistant SA isolates were collected out of patient samples admitted to the Laboratory of Institute of Public Health of Canton Sarajevo, Bosnia and Herzegovina.

We selected a representative sample of 100 strains, eliminating the "copy" strains (strains from the same patient at different places and those that are repeated). Among one hundred MRSA isolates 49 (49\%) were from males and 51 (51\%) from females. There was no significant sex difference in MRSA distribution. The mean age of examinees was $10.9 \pm 18.7$ years. Of the total number of isolates largest number of isolates included in our study was isolated from nasal swabs or nasopharyngeal (41\%), 35.0\% isolates were from skin swabs, $7 \%$ from pustules, $5 \%$ from wound infections, $3 \%$ from throat swabs, $3 \%$ from ear swabs, and $6 \%$ from various other body sites.

All MRSA isolates were resistant to the $\beta$-lactam antibiotics tested, i.e. penicillin, oxacillin cefoxitin, and all isolates were susceptible to trimethoprim - sulphamethoxazole, rifampicin, fusidic acid, linezolid, and vancomycin; $68 \%$ of MRSA isolates were resistant to erythromycin, $5 \%$ to clindamycin, $5 \%$ to gentamicin, and $4 \%$ to ciprofloxacin (Table 1 ).
Table 1. Antimicrobial susceptibility patterns $(N=100)$

\begin{tabular}{|l|c|c|}
\hline \multirow{2}{*}{ Penicillin } & Resistant & Sensitive \\
\cline { 2 - 3 } & $\mathbf{n}(\%)$ & $\mathbf{n}(\%)$ \\
\hline Oxacillin & $100(100)$ & 0 \\
\hline Cefoxitin & $100(100)$ & 0 \\
\hline Erythromycin & $100(100)$ & 0 \\
\hline Clindamycin & $68(68)$ & $32(32)$ \\
\hline Sulfamethoxazole + trimethoprim & $5(5)$ & $95(95)$ \\
\hline Rifampin & 0 & $100(100)$ \\
\hline Fusidic acid & 0 & $100(100)$ \\
\hline Gentamicin & 0 & $100(100)$ \\
\hline Ciprofloxacin & $5(5)$ & $95(95)$ \\
\hline Linezolid & $4(4)$ & $96(96)$ \\
\hline Vancomycin & 0 & $100(100)$ \\
\hline
\end{tabular}

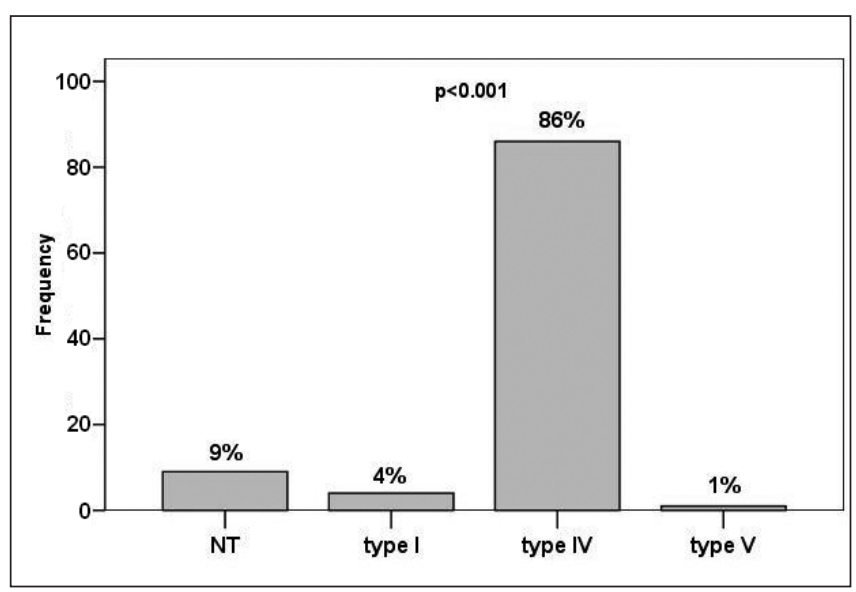

Fig. 1. SCCmec types distribution among MRSA isolates.

NT - non-typeable

The most prevalent SCCmec element was type IV (86\%), followed by SCCmec I (4\%), and SCCmec type V (1\%). Nontypeable strains were also found in $9 \%$ of cases. No isolates with $\mathrm{SCCmec} \mathrm{III} \mathrm{were} \mathrm{found} \mathrm{during} \mathrm{this} \mathrm{analysis.} \mathrm{For} \mathrm{the} \mathrm{analysis} \mathrm{of}$ categorical variables was used chi-square test. A summary of the results is presented in Figure 1.

A total of $24 \%$ of isolates had positive expression of PVL genes, while $76 \%$ showed a statistically significantly greater negative expression of PVL genes $(\mathrm{p}<0.05)$.

Of 24 PVL positive isolates, 16 were recovered from samples skin changes swabs, while 8 were from nasal swabs (Table 2).

Table 2. Different MRSA strains in relation to $P V L$ gene $(N=100)$

\begin{tabular}{|c|c|c|c|c|c|c|c|c|c|c|}
\hline \multirow{3}{*}{ MRSA strains } & \multicolumn{9}{|c|}{ Source of specimens } & \multirow{3}{*}{$\begin{array}{l}\text { Total } \\
\mathrm{n}(\%)\end{array}$} \\
\hline & \multicolumn{8}{|c|}{ Swabs } & \multirow{2}{*}{ Sputum } & \\
\hline & Nose & Throat & Ear & Eye & Umbilicus & Wound & Skin & Pustule & & \\
\hline PVL positive & 8 & & & & & & 16 & & & $24(24)^{*}$ \\
\hline PVL negative & 33 & 3 & 2 & 2 & 1 & 5 & 24 & 5 & 1 & $76(76)$ \\
\hline
\end{tabular}




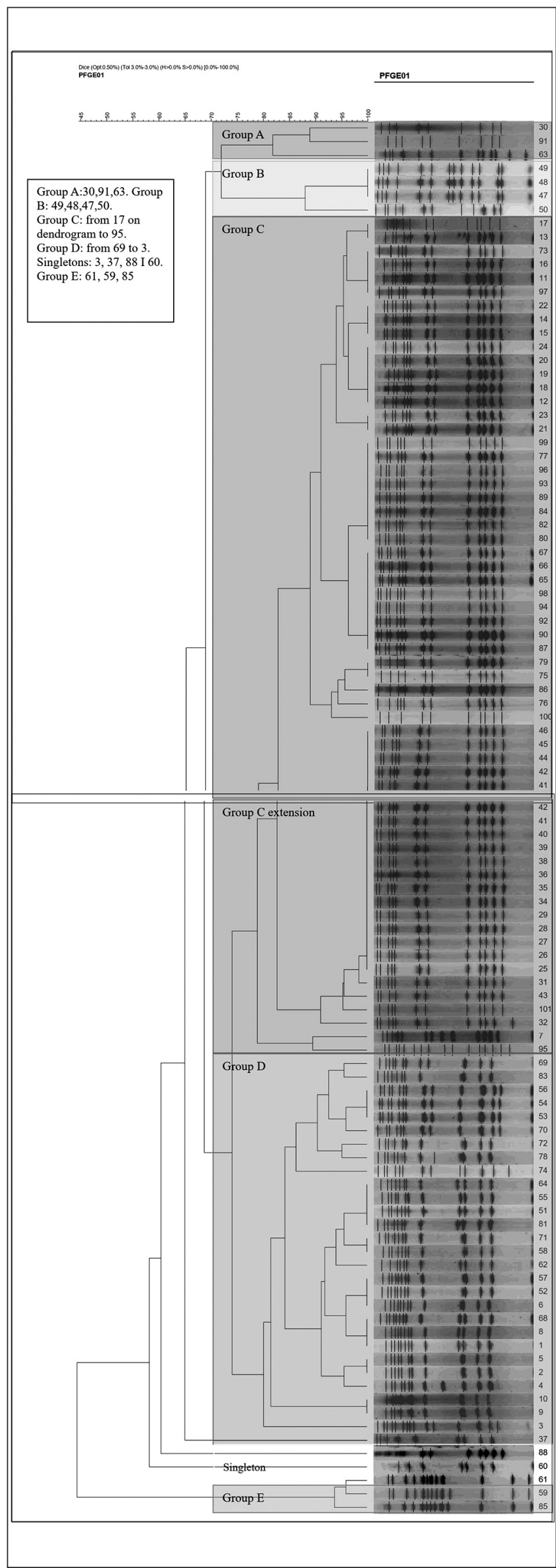

Fig. 2. PFGE dendrogram of methicillin-resistant Staphylococcus aureus isolates included in the study.
After the PFGE analysis, the isolates were placed together in five similarity groups: A-E. The largest number of isolates $(87 \%)$ belonged to one of two groups: $\mathrm{C}(60 \%)$ and $\mathrm{D}(27 \%)$.

Group A included three isolates, which were SCCmec type IV. In this group there were not PVL positive isolates. Group B included four isolates, where three isolates were SCCmec type I, while one was SCCmec type IV. There were not PVL positive isolates in this group as well. Group C represents the largest group in our PFGE analysis. All isolates of this group belong to SCCmec type IV. In this group of $28,33 \%$ of isolates were PVL positive. Group D included 27 isolates, of which three were non-typeable, and the rest belong also to SCCmec type IV. In this group, only two isolates were PVL positive. Group E included 3 isolates, SCCmec type I, IV and V. These three isolates were not PVL positive. Three isolates were singletons, i.e., not similar to any of the other strains.

One of 100 isolates could not be typed using PFGE, either owing to difficulties with lysis of the bacteria or the digestion of the DNA. A total of 5 similarity groups (A to E) were found and two of these, $\mathrm{C}$ and $\mathrm{D}$, were classified as major similarity groups (Figure 2). Similarity group C harboured 60 of 100 isolates, whereas similarity group D harboured 27 of 100 isolates. In both groups $\mathrm{C}$ and $\mathrm{D}$, SCCmec type IV was predominant (i.e. $60 \%$ and $88.8 \%$, respectively).

\section{DISCUSSION}

Our study showed, for the first time in Bosnia and Herzegovina, the emergence and molecular detection communityassociated methicillin resistant Staphylococcus aureus. Since many definitions for CA-MRSA exist (17), general and international agreement has been reached on a universal definition of CA-MRSA. The Center for Disease Control and Prevention (CDC) defines CA-MRSA as strains isolated in an outpatient setting, or from patients within 48 hours of hospital admission. Furthermore, these patients must have no medical history of MRSA infection or colonisation, and no medical history in the past year of either hospitalisation (e.g. surgery), admission to a nursing home, or dialysis. Moreover, the patient should not have permanent in-dwelling catheters or medical devices that pass through the skin.

These infections are commonly referred to as communityacquired MRSA. CA-MRSA infections are endemic and regarded as a major public health care concern worldwide (18), CA-MRSA demonstrates resistance to penicillin and methicillin but remains susceptible to non- $\beta$-lactam antimicrobials, such as clindamycin, sulfamethoxazole-trimethoprim, and tetracyclines. Our results confirmed such trends and indicated lower resistances to clindamycin, ciprofloxacin, erythromycin, and gentamicin (17). Additionally, all isolates were susceptible to sulfamethoxazole-trimethoprim, rifampicin, fusidic acid, linesolide, and vancomycin. Results in region countries (e.g. Croatia) were in concordance with our results regarding antimicrobial susceptibility (19). General health decline with age, higher antibiotic regimens, lack of proper education, and less frequent visits to physicians are influential factors in the rapid evolution of antibiotic-resistant species.

PVL is SA specific exotoxin and its genes have been demonstrated primarily among CA-MRSA (20). 
Several studies showed that PVL genes were found in $<5 \%$ of $S$. aureus isolates worldwide, whereas the rates of carriage of the PVL toxin gene are $>75 \%$ for CA-MRSA strains (21). However, in our study was shown $24 \%$ of PVL positive from CA-MRSA. This big different rate of PVL positive from CA-MRSA might be caused by the fact that of 100 representative samples subjected to molecular analysis was only $35 \%$ isolates of the skin. Furthermore, if we show only skin isolates in this study, there are $45.71 \%$ (16 PVL+) of positive PVL from CA-MRSA.

High rates of PVL-producing MRSA were reported in previous studies in the general population, in several countries such as the USA (22) and Tunisia (23). On the contrary, lower rates or no PVL positive MRSA were reported in other countries, e.g. 15\% in the Netherlands (24), $0.7 \%$ in Japan (25), and near to zero in some English hospitals (26).

For detection of PVL and methicillin-resistance (mecA) genes represent a new tool to aid the early identification of CA-MRSA isolates (27). Using multiplex PCR, both of two parameters, PVL and mecA did not find from our 76 samples but were separately detected. The identification of $S$. aureus isolates carrying PVL genes is an important first step in controlling the virulence. The combination of both PVL and mecA shows very potent virulence with resistance to antibiotics such as oxacillin. Consequently, it is very important to identify serious and harmful $S$. aureus from both detection of PVL and mecA. However, several studies are shown such as PVL (-) MSSA, PVL (+) MSSA, PVL (-) MRSA and PVL (+) MRSA (21-26).

The resistance of bacteria is caused by acquisition of the methicillin resistance gene mecA, carried by the staphylococcal cassette chromosome mec ( $\mathrm{SCCmec}$ ). By the time the Guidelines for the classification of SCCmec elements were prepared, eleven SCCmec types have been described for SA (28).

SCCmec types IV and V elements generally carried by CAMRSA strain were reported (29). In our study, $86 \%$ isolates carried the SCCmec type IV complex that included all PVL positive strains. Only one isolate carried the SCCmec type V complex but it was PVL negative strain. Comparison grouping by PFGE and SCCmec typing was used to determine whether our strains belonged to HA- or CA-MRSA strains. The majority of isolates in PFGE group $\mathrm{C}$ represented the largest group in our PFGE analysis. All isolates of this group belonged to SCCmec type IV. In this group of $28,33 \%$ of isolates were PVL positive. Group D included 27 isolates, of which three non-typeable, and the rest belong also to SCCmec type IV. In this group, only two isolates were PVL positive.

Boyle-Vavra and Daum (18) found that 50 to $93 \%$ of the $S$. aureus strains causing primary skin infections produce PVL, in accordance with the results of our research. Furthermore, a study by Gillet et al. (8) demonstrated a strong association between PVL and necrotizing pneumonia in healthy children and young adults. From these studies, the authors hypothesized that the propensity of many CA-MRSA strains to cause severe skin and soft tissue infections and, occasionally, necrotizing pneumonia is due to their ability to produce PVL (8). Consequently, community-based healthcare providers such as family doctors play a key role in transmission prevention. This case highlights the importance of increased awareness when dealing with recurrent skin infections affecting either individuals or individuals closely associated with each other, as in a family setting.
Our study has several limitations. First, the relatively small sample of respondents and short period of research. Second, the examination did not include all microbiological laboratories in the country. There is a need for national monitoring of the spread of existing epidemic strains, as well as the emergence of new strains, which would allow the inclusion of Bosnia and Herzegovina in an international network of monitoring resistant bacteria.

\section{CONCLUSION}

Using combination techniques, we were able to investigate the origin and genetic background of the strains. PFGE analysis revealed two large, genetically related groups of strains consisting of 87 isolates. Our results suggest failure to apply the screening policy, and a lack of knowledge about multiresistant MRSA strains. This study showed the local epidemiological situation which should be the basis of antimicrobial empiric therapy for non-hospitalized patients.

\section{Conflict of Interests}

None declared

\section{Adherence to Ethical Standards}

Ethical approval was received from the Ethics Committee of the University School of Medicine, Zagreb, Croatia (No.04-77/2010-62), and Faculty of Medicine, University of Sarajevo, Bosnia and Herzegovina (No.0305-18501).

\section{Declaration of Authorship}

VR and AB performed literature search, research organization and coordination, data collection, data organization, and wrote the manuscript. MA performed the part of statistical analysis and performed manuscript review, SMV performed data interpretation and manuscript review, DR performed the study design.

\section{REFERENCES}

1. Mulcahy ME, Geoghegan JA, Monk IR, O'Keeffe KM, Walsh EJ, Foster TJ, et al. Nasal colonisation by Staphylococcus aureus depends upon clumping factor $\mathrm{B}$ binding to the squamous epithelial cell envelope protein loricrin. PLoS Pathog. 2012 Dec;8(12):e1003092. doi: 10.1371/journal. ppat. 1003092 .

2. Udo EE. Community-acquired Methicillin-resistant Staphylococcus aureus: the new face of an old foe? Med Princ Pract. 2013;22(1):20-9.

3. Moellering RC Jr. MRSA: the first half century. J Antimicrob Chemother. 2012;67(1):4-11.

4. Bonesso MF, Marques SA, Cunha MLRS. Community-acquired methicilin-resistant Staphylococcus aureus (CA-MRSA): molecular background, virulence, and relevance for public health. J Venom Anim Toxins. 2011;17(4):378-86.

5. Fuda C, Suvorov M, Vakulenko SB, Mobashery S. The Basis for resistance to b-lactam antibiotics by penicillin binding protein $2 \mathrm{a}$ of methicillinresistant Staphylococcus aureus. J Biol Chem. 2004;279:40802-6.

6. Shore AC, Deasy EC, Slickers P, Brennan G, O'Connell B, Monecke S, et al. Detection of staphylococcal cassette chromosome mec type XI carrying highly divergent mecA, mecI, mecR1, blaZ, and ccr genes in human clinical isolates of clonal complex 130 methicillin-resistant Staphylococcus aureus. Antimicrob Agents Chemother. 2011 Aug;55(8):3765-73.

7. Lindsay JA. For CA-MRSA, how much PVL is too much? Microbiology. 2009;155(11):3473-4.

8. Gillet Y, Issartel B, Vanhems P, Fournet JC, Lina G, Bes M, et al. Association between Staphylococcus aureus strains carrying gene for 
Panton-Valentine leukocidin and highly lethal necrotising pneumonia in young immunocompetent patients. Lancet. 2002;359(9308):753-9.

9. Varshney AK, Martinez LR, Hamilton SM, Bryant AE, Levi MH, Gialanella P, et al. Augmented production of Panton-Valentine leukocidin toxin in methicillin-resistant and methicillin-susceptible Staphylococcus aureus is associated with worse outcome in a murine skin infection model. J Infect Dis. 2010 Jan 1;201(1):92-6.

10. Oliveira DC, de Lencastre H. Multiplex PCR strategy for rapid identification of structural types and variants of the mec element in methicillin-resistant Staphylococcus aureus. Antimicrob Agents Chemother. 2002;46(7):2155-61.

11. Budimir A, Deurenberg RH, Plecko V, Vink C, Kalenic S, Stobberingh EE. Molecular characterization of methicillin-resistant Staphylococcus aureus bloodstream isolates from Croatia. J Antimicrob Chemother. 2006;57(2):331-4.

12. Lina G, Piemont Y, Godail-Gamot F, Bes M, Peter MO, Gauduchon V, et al. Involvement of Panton-Valentine leukocidin-producing Staphylococcus aureus in primary skin infections and pneumonia. Clin Infect Dis 1999;29(5):1128-32.

13. Murchan S, Kaufmann ME, Deplano A, de Ryck R, Struelens M, Zinn $\mathrm{CE}$, et al. Harmonization of pulsed-field gel electrophoresis protocols for epidemiological typing of strains of methicillin-resistant Staphylococcus aureus: a single approach developed by consensus in 10 European laboratories and its application for tracing the spread of related strains. J Clin Microbiol. 2003;41(4):1574-85

14. Tenover FC, Arbeit RD, Georing RV, Mickelsen PA, Murray BE, Persing $\mathrm{DH}$, et al. Interpreting chromosomal DNA restriction patterns produced by pulsed-field gel electrophoresis: criteria for bacterial strain typing. J Clin Microbiol. 1995;33(9):2233-9.

15. Koreen L, Ramaswamy SV, Graviss EA, Naidich S, Musser JM, Kreiswirth BN. Spa typing method for discriminating among Staphylococcus aureus isolates: implications for use of a single marker to detect genetic micro- and macrovariation. J Clin Microbiol. 2004;42(2):792-9.

16. Harmsen D, Claus H, Witte W, Rothgänger J, Claus H, Turnwald D, et al. Typing of methicillin-resistant Staphylococcus aureus in a university hospital setting by using novel software for spa repeat determination and database management. J Clin Microbiol. 2003;41(12):5442-8.

17. Deurenberg RH, Vink C, Kalenic S, Friedrich AW, Bruggeman CA, Stobberingh EE. The molecular evolution of methicillin-resistant Staphylococcus aureus. Clin Microbiol Infect. 2007;13(3):222-35.

18. Boyle-Vavra S, Daum RS. Community-acquired methicillin-resistant Staphylococcus aureus: the role of Panton-Valentine leukocidin. Lab Invest. 2007;87(1):3-9.

19. Budimir A, Payerl Pal M, Bošnjak Z, Mareković I, Vuković D, Roksandić Križan I, et al. Prevalence and molecular characteristics of methicillin resistant Staphylococcus aureus strains isolated in a multicenter study of nursing home residents in Croatia. Am J Infect Control. 2014;42(11):1197202
20. Sanchini A, Del Grosso M, Villa L, Ammendolia MG, Superti F, Monaco $\mathrm{M}$, et al. Typing of Panton-Valentine leukocidin-encoding phages carried by methicillin-susceptible and methicillin-resistant Staphylococcus aureus from Italy. Clin Microbiol Infect. 2014 Nov;20(11):O840-6.

21. Hamilton SM, Bryant AE, Carroll KC, Lockary V, Ma Y, McIndoo E, et al. In vitro production of Panton-valentine leukocidin among strains of methicillin-resistant Staphylococcus aureus causing diverse infections. Clin Infect Dis. 2007;45(12):1550-8.

22. Fortunov RM, Hulten KG, Hammerman WA, Mason EO Jr, Kaplan SL. Community-acquired Staphylococcus aureus infections in term and nearterm previously healthy neonates. Pediatrics. 2006;118(3):874-81.

23. Ben Nejma M, Mastouri M, Bel Hadj Jrad B, Nour M. Characterization of ST80 Panton-Valentine leukocidin-positive community-acquired methicillin-resistant Staphylococcus aureus clone in Tunisia. Diagn Microbiol Infect Dis. 2013;77(1):20-4.

24. Wannet WJ, Spalburg E, Heck ME, Pluister GN, Tiemersma E, Willems RJ, et al. Emergence of virulent methicillin-resistant Staphylococcus aureus strains carrying Panton-Valentine leucocidin genes in The Netherlands. J Clin Microbiol. 2005 Jul;43(7):3341-5.

25. Yanagihara K, Araki N, Watanabe S, Kinebuchi T, Kaku M, Maesaki S, et al. Antimicrobial susceptibility and molecular characteristics of 857 methicillin-resistant Staphylococcus aureus isolates from 16 medical centers in Japan (2008-2009): nationwide survey of community-acquired and nosocomial MRSA. Diagn Microbiol Infect Dis. 2012;72(3):253-7.

26. Shallcross LJ, Williams K, Hopkins S, Aldridge RW, Johnson AM, Hayward AC. Panton-Valentine leukocidin associated staphylococcal disease: a cross-sectional study at a London hospital, England. Clin Microbiol Infect. 2010;16(11):1644-8.

27. McClure JA, Conly JM, Lau V, Elsayed S, Louie T, Hutchins W, et al. Novel multiplex PCR assay detection of the staphylococcal virulence marker PantonValentine leukocidin genes and stimultaneous discrimination of methicillin-susceptible from-resistant staphylococci. J Clin Microbiol. 2006 Mar;44(3):1141-4.

28. International Working Group on the Classification of Staphylococcal Cassette Chromosome Elements (IWG-SCC). Classification of staphylococcal cassette chromosome mec (SCCmec): guidelines for reporting novel SCCmec elements. Antimicrob Agents Chemother. 2009;53(12):4961-7.

29. Tristan A, Michele Bes, Meugnier H, Lina G, Bozdogan B, Courvalin $\mathrm{P}$, et al. Global distribution of Panton-Valentine leukocidin-positive methicillin-resistant S. aureus, 2006. Emerg Infect Dis. 2007;13(9):594600

Received August 3, 2016

Accepted in revised form December 14, 2018 